\title{
Quantitative evaluation of formal based synthesis in ASIC design
}

\author{
G. Bezzi, M. Bombana, P. Cavalloro, S. Conigliaro, G. Zaza \\ ITALTEL DRSC Settimo Milanese (ITALY)
}

\begin{abstract}
Formal based synthesis allows design space exploration to identify optimized implementations conforming to the initial abstract specification. We propose to exploit the synergies between formal synthesis (at high level of abstraction) and logic synthesis (at lower levels of abstraction). In this way a two-fold goal is reached: quantitative figures are provided as a measure of the applicability of formal reasoning in the design process, and the good integration of the two phases in a unified design flow is demonstrated. Users' benefits include both improved quality of the design process (reduced time-to-market) and improved reliability of the final products (increased competitive profile).
\end{abstract}

\section{Introduction}

The industrial interest in the application of formal methods to the design of complex ASICs is noteworthy. Design methodologies have been proposed ([8],[15],[16],[17], $[18],[23],[24])$ and commercial tools are now available ([9],[19]) to partially support the design practice with the power of formal reasoning. In this paper we focus on the application of high-level formal based synthesis ([3],[14]), that aims at improving the management of the first phases of the design process, addressing both the specification phase and the following partitioning steps at the architectural and/or scheduling/allocation levels. In this way a coherent and sound approach to the initial transformations of the design entities is provided, covering a phase of the design practice where automatic tools are still not widely used.

To apply this approach to real industrial practice ([12],[13]), some pre-requisites must be satisfied. One of them involves the definition of a homogeneous design flow able to link in a coherent way the results coming from the application of high-level formal synthesis with the standard techniques and tools of the lower levels of the design flow. In fact the gap existing between this theoretical approach and the present industrial design environments remains relevant: while the former improves the reasoning capabilities stressing abstraction and generality, the latter are mainly capable of dealing with completely instantiated specifications of RTL descriptions.

In section 2 a formal based synthesis tool (LAMBDA/DIALOG [9]) is introduced for design space exploration as a 'stand-alone' advisor. In this phase the designer focuses on the search for an 'optimal' solution in terms of architectural choices or scheduling and allocation strategies. In section 3 this design phase is integrated into a homogeneous design flow in accordance with the users' requirements. Experimental results, obtained applying different strategies are compared and design guidelines are extrapolated to increase the efficiency of the global process. Considerations on the still existing problems and some hints on the future of this research are addressed in the conclusion.

\section{Formal synthesis: the use of a 'stand-alone' high-level advisor}

Formal methods have been applied in the design process at different abstraction levels and with different goals ([1],[7]). LAMBDA/DIALOG allows exploration of the early design space formally guaranteeing the transformations of an abstract behavioral specification of the device into mixed (behavioral/structural) representations [2].

(*) This research activity is part of the ESPRIT II/III Projects n. 5020 and 6128. 


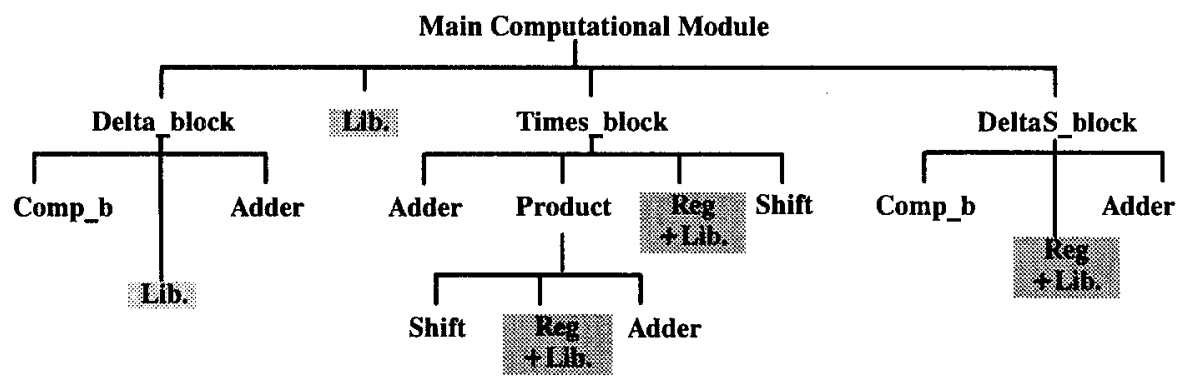

Fig. 1. Hierarchic representation of the modules of the Computational Block.

The tool differs substantially from other high-level behavioral synthesis tools. First, it is the only commercial one applying formal methods to guarantee the correctness of transformations between different description levels, and second it allows extensive user's interaction. The main benefit of the DIALOG interface [9] is to assist the designer in the process of step-wise transforming the starting specification into the chosen implementation. The supported specification language (ML [9]) is a functional language rather different from the most used hardware description languages, as the 'defacto' standard VHDL [16].

\subsection{The initial exploration of the design space}

The synthesis process of a complex device is managed applying a 'divide and conquer' strategy: our test case [6] consists of 4 levels of hierarchy including 32 netlists and approximately 330 instances of user-defined modules. The low level modules of this device partially overlap the test cases that have been proposed as benchmarks [20]. The application of the formal techniques are shown to these low-level modules. The same considerations are valid for modules of higher level and for architectural partitionings, without any theoretical difference.

The comparison of implementation alternatives is particularly useful for those modules that are usually critical in terms of area overhead and timing properties, such as those performing the computations. The main Computational Module (Fig. 1) is based on the implementation of three sub-modules and some random logic. A preliminary analysis shows that the efficient combination of $\mathrm{N}$-bit adders (Adder blocks) and $\mathrm{N}$-bit multipliers (Product blocks)strongly influences the values of the physical parameters of the final implementation and the structure of the associated control blocks. As a consequence, the choices applied when designing the Computational Module are crucial.

Different specifications and implementations have been analyzed in LAMBDA in order to identify the most efficient solution for the computational sub-modules focusing on the implementation of the Product blocks (third hierarchic level of partitioning). The considered parameters were synchronous vs. asynchronous specification, parallelism of operations, granularity of the basic operations in terms of bits. The most naïve approach uses the maximum number of resources: the product is implemented through sequential additions and shift operations on the input data.

\subsection{The re-use strategy and the search for the best solution}

A solution to decrease the area is reached applying the re-use strategy to the sub-elements (Adder and Shift blocks) appearing in the Product netlist. This strategy allows the reduction of the area (the same component is used for more than one computation cycle) and involves the introduction of some registers for synchronization purposes. 
Cycles are defined easily in LAMBDA applying a 're-use' tactic followed by a 'scheduling and allocation' tactic to introduce synchronization elements. The selection of the computational blocks to be re-used is done by the designer.

Moreover the computation on a N-bit data can be partitioned into two N/2-bit operations, with the introduction of an adder for the combination of the partial results. In this way the pure sequential procedure applied in the initial specification is substituted by the introduction of a certain level of parallelism, able to reduce the global clock phase of the computation. Applying both techniques and different levels of 're-use' in alternative implementations produces a wide range cases of different characteristics of area and timing: a single adder and a single shift block, two couples of each and finally four couples of each (Table 1, case II-III-IV). The time spent by the designer to produce these alternative representations is very small (a few hours). The control micro-code is also generated automatically by the tool.

The comparison between the different solutions is based on qualitative parameters: the number of components added for the control overhead is given, but no exact indication can be extracted on the relative increase of area on the total, or on the relative variations in the different cases. A more quantitative evaluation can be based on the results of logic synthesis.

\section{The integration of formal synthesis into a design environment}

The integrated approach to design, extensively applied nowadays, doesn't exclude the usefulness of stand-alone advisers on special aspects of the design activity. Anyway, when large-scale exploitation is foreseen, the identification of a global design flow is the winning element for the introduction of new tools into the consolidated design practice.

No single vendor provides tools to cover the whole range of requirements and needs of the design process. As a consequence a multi-vendor design environment is implemented [10] assembling the best tools on the market to solve specific tasks. CAD frameworks [5] provide the unified view and facilities to implement customized features for each proprietary design flow. Different levels of integration into a CAD framework are possible, i.e. a strong integration or a weak integration, usually called also encapsulation. The former implies for a tool the complete accessibility of the internal data-base of the framework, while the latter implies no data-base shating and the interaction between the proprietary tool and the framework is accomplished in terms of shared files. The encapsulation path is usually followed. The requirements imposed on the tools are rather loose [22]. LAMBDA and the implemented interfaces satisfy these requirements and their encapsulation constitutes the prototype environment used to derive the results presented in the next sections.

\subsection{The extended design flow}

A design flow including formal synthesis and logic synthesis handles the substantial syntactic/semantic representation gaps existing between the levels of abstraction at which the tools operate. Formal synthesis tools operate abstractly and implementation details are left unspecified on purpose. On the contrary logic synthesis tools need specifications in a restricted subset of VHDL ([4],[11]) where all the implementation details have been correctly instantiated.

The handling of the instantiation mechanism, corresponding to a design level transformation from the most abstract to the most implementation-oriented description, is managed with the introduction of a transformational module supported by libraries of functional blocks, described in VHDL suitable for logic synthesis. 
Commercial VHDL data-bases [21] support actively the development of this environment. From an operative point of view, three main design phases are managed by the designer. The first phase prepares the environment definition and the interface instantiation of the entities, as required by the VHDL description. The second phase identifies the standard library bloeks in the netlist, for which the VHDL description is included in the functional parametric library. The user specifies the characteristics of each block in order to allow the correct instantiation. The third phase involves the identification of the user-defined modules, for which a behavioral VHDL description is not pre-defined. The resulting design environment has been tested only for the development of prototypes and re-design of already existing devices.

\subsection{Experimental results and evaluation of physical parameters}

The final result of the first half of the design flow of Fig. 2 is a VHDL netlist. A mixed VHDL simulation controls the correctness of the transformations. The numeric figures obtained applying the second half of the design flow to the examples considered in section 2, are shown in Table 1. The first two rows indicate for each implementation, if the $\mathrm{N}$-bit multiplication has been partitioned, and how many adders and shifts have been inserted to manage the partitioning. The third row gives the number of equivalent gates for each implementation and the fourth the timing parameters. These values can be compared with the initial design constraints. Solution I is satisfactory in terms of equivalent gates, but its timing performances are too elevated; all the sequential components

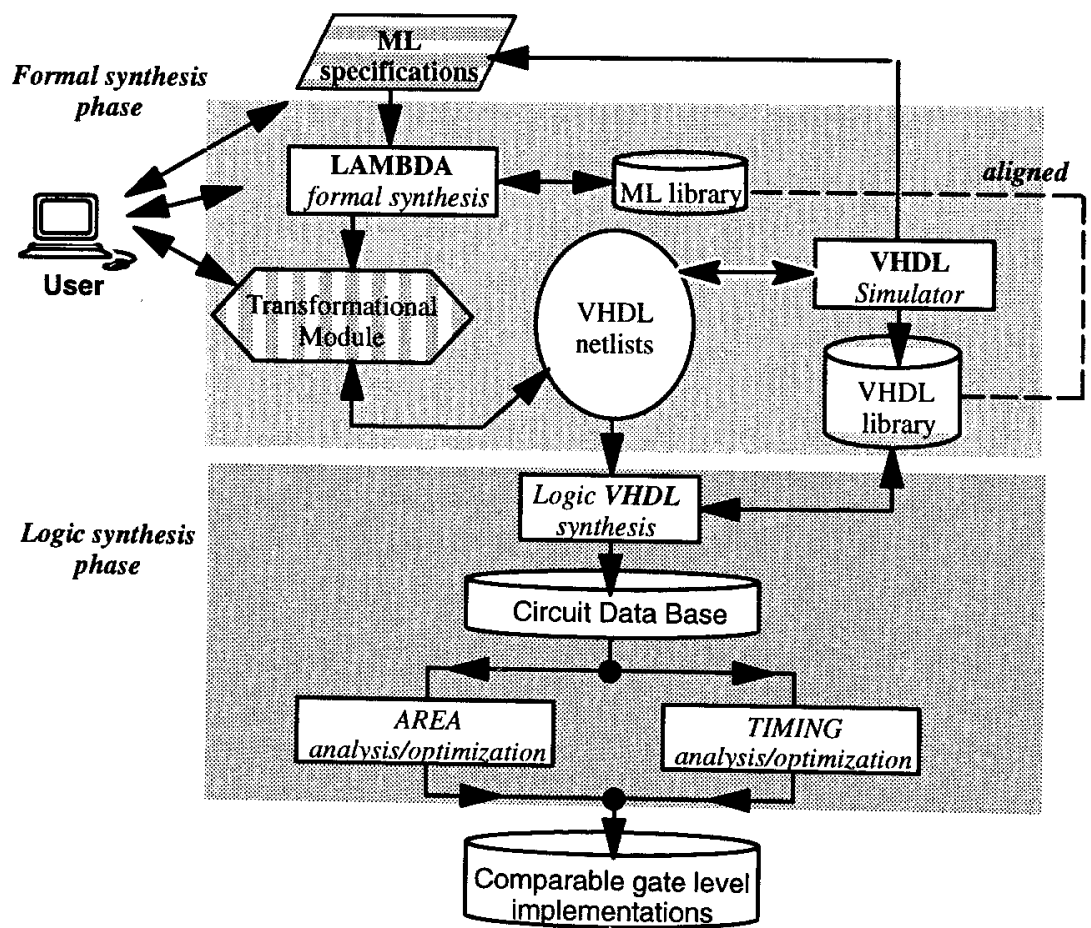

Fig. 2. The design flow. 
have been stripped off, with the effect of reducing the area but transforming the circuit into a sort of asynchronous component.

\begin{tabular}{|c|c|c|c|c|c|c|c|}
\hline The cases: & 17 & II & .111 & IV & V & y & (111 \\
\hline Conputational Medile & async. & re-use & re-use & re-use & re-use & re-use & re-use \\
\hline $\begin{array}{l}\text { Times blodk } \\
\text { 4 i products (bit dim) }\end{array}$ & $\frac{1}{(16-b i t)}$ & $\frac{2}{(8-b i t)}$ & $\stackrel{2}{2}$ & $\begin{array}{c}2 \\
(8-b i t)\end{array}$ & $\begin{array}{c}4 \\
(4-b i t)\end{array}$ & $\begin{array}{c}4 \\
(4-b i t)\end{array}$ & $\begin{array}{c}4 \\
(4-b i t)\end{array}$ \\
\hline 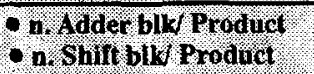 & $\overline{-}$ & 1 & 2 & $\begin{array}{l}4 \\
4\end{array}$ & $\begin{array}{l}1 \\
1\end{array}$ & 2 & $\begin{array}{l}4 \\
3\end{array}$ \\
\hline (numb. of Equiv. gates) & 4712 & 10927 & 5612 & 3696 & 5623 & 3824 & 4645 \\
\hline $\begin{array}{l}\text { Area Optim. } \\
\text { (numb. of Equiv. gates) }\end{array}$ & 4697 & 6552 & 3846 & 3027 & $\mathbf{3 7 7 2}$ & 3342 & 4442 \\
\hline (Glock (n) \& e gates) & $\begin{array}{c}148 \\
4697\end{array}$ & $\begin{array}{c}72 \\
6552\end{array}$ & $\begin{array}{c}84 \\
3846\end{array}$ & $\begin{array}{c}118 \\
3027\end{array}$ & $\begin{array}{c}90 \\
3772\end{array}$ & $\begin{array}{c}89 \\
3342\end{array}$ & $\begin{array}{c}111 \\
4442\end{array}$ \\
\hline $\begin{array}{l}\text { Tining Optín. } \\
\text { (Clocl (ns) \& e gates) }\end{array}$ & $\begin{array}{c}95 \\
5375\end{array}$ & $\begin{array}{c}44 \\
6736\end{array}$ & $\begin{array}{c}48 \\
4076\end{array}$ & $\begin{array}{c}67 \\
3362\end{array}$ & $\begin{array}{c}61 \\
3984\end{array}$ & $\begin{array}{c}60 \\
3690\end{array}$ & $\begin{array}{c}69 \\
5130\end{array}$ \\
\hline
\end{tabular}

Table 1. Summary of the characteristics of the different implementations

Table 1 shows significant differences between the other implementations differing for the value of re-use. Case II is characterized by the greatest amount of area overhead but it is the fastest in terms of clock cycle; on the other hand, case IV has the most reduced area but the timing performances are the worst of this group. Anyway this implementation gives better results than case I for both parameters. The explanation of these results lies in the different delay characteristics of the computation caused by the adopted re-use strategy.

Further refinements are obtained increasing the level of parallelism in decomposing the 16-bit multiplication element into four 4-bit parallel multiplications. The implementation of this case has been done considering, as in the previous case, alternative re-use strategies (cases V-VI-VII). The figures associated with these last implementations show that the parallelism introduced at the multiplication level generates a nonlinear dependence between area and timing parameters. The last two rows of Table 1 show the results of the optimization phase on the seven alternative implementations. The comparison of these figures with those of the previous rows of Table 1 shows that in some cases the gain is close to a factor of two.

None of the automatically obtained implementations is more efficient than the one designed and optimized 'by hand' by an expert. senior designer.

General guidelines may be abstracted. The synthesis process of computational modules in LAMBDA should be performed reducing as much as possible the number of synchronization components responsible of increased area overhead. This action must be coupled with an optimization of the VHDL functional library. The behavior of components must be written avoiding the use of statements that involve a complex hardware implementation in the logic synthesis phase. The re-use strategy is useful in order to reduce both area overhead and timing parameters. But to be effective a medium coefficient of re-use must be applied.

\section{Conclusions}

In this paper we have described the prototype of an innovative design methodology with associated tools supporting both formal reasoning and standard design techniques 
in a unified design flow. This new approach can be applied to the design of complex ASICs, to increase the global quality of the produced devices coupled with a decreased time-to-market. From this research activity suggestions have been collected to make the tools more user-friendly and more accepted to the users' community.

An extended engineering activity must be pursued in future, before applying this methodology in the current design practice, to make it sound and reliable. An extended test of the involved tools must verify their ability to cope with different classes of applications and with the increasing complexities of the devices to be designed.

\section{References}

1. F. Anceau: Formal verification in industrial environment, Workshop on Formal Methods, L'Aquila, (1989)

2. M.Fourman, E. Mayger: Formally Based System Design - Interactive Hardware scheduling, G. Musgrave, U.Lauther (eds), VLSI '89, Elsevier (1989)

3. S. Finn, M. Fourman, M. Francis, R. Harris: Formal System design - Interactive Synthesis based on Computer-Assisted Reasoning, Proc. IFIP WG 10.2, 10.5 Workshop, North Holland, (1990)

4. D. L. Perry: VHDL, McGraw-Hill, Inc. (1991)

5. Mentor Graphics: Getting started with Falcon Framework, (1991)

6. ESPRIT II Project n. 5020: Technical Report WP1.2-1, (1991)

7. F. Anceau: Panel on formal methods in hardware design, 10th Int. Symp. on Computer Hardware Description Languages, Marseille, (1991)

8. L. Claesen, M. Genoe, E. Verlind, F. Proesmans, H. De Man: SFGTracing: a methodology of design for Verifiability, Proc. Adv. Res. Workshop on Correct Hardware Design Methodologies, Turin, (1991)

9. AHL: Lambda Reference Manual - Version 4.1, London (1992)

10.M. Miserandino: ITL_TOOLKIT 1.0, Italtel Sit, Milano (1992)

11.C. Costi: A VHDL subset definition for simulation and synthesis in the Italtel environment, ITALTEL Technical Report, (1992)

12.G. Gorla: L'automazione del progetto di sistema nell'industria, Workshop on Specification and Synthesis of Digital Systems, CEFRIEL (1992)

13.M. Bombana, P. Cavalloro, G. Zaza: Specification and formal synthesis of digital circuits, Proc. IFIP TC10/WG10.2 Workshop, North Holland, (1992)

14.R.B. Hughes, G. Musgrave: Design Flow Graph Partitioning, HOL '92, IMEC Leuven Belgium, (1992)

15.R. Schlör, W. Damm: Specification and verification of system-level hardware designs using timing diagrams, EDAC '93, (1993)

16.S. Olcoz, J. M. Colom: Toward a Formal semantics of IEEE Std. VHDL 1076, EURO-DAC'93, Hamburg (1993)

17.C. Bolchini, M. Bombana, P. Cavalloro, C. Costi, F. Fummi, G. Zaza: A design methodology for the correct specification of VLSI systems, Euromicro '93, Barcelona, (1993)

18. T. Robles Valladares, A. Marín López, C. Delgado Kloos, T. de Miguel Moro, G. Rabay Filho: Automatic Hardware Implementation of Formal Specifications, III Jornadas de Concurrencia, Gandia, (1993)

19.CLSI Solutions: VFormal, (1993)

20.Benchmark circuits for hardware verification, Univ. of Karlsruhe, (1993)

21.LEDA: VHDL System, Meylan (1993)

22.ESPRIT III n. 6128: The FORMAT Design Methodology, Tech. Rep., (1994)

23.W. Grass, M. Mutz, W.D. Tiedemann: High Level Synthesis Based on Formal Methods, Euromicro '94, (1994)

24.K. L. McMillan: Fitting Formal methods into the Design Cycle, DAC '94, San Diego (1994) 